\section{Paroxysmal Tachycardia in Children}

It is important to be aware that babies and small children may suffer from paroxysmal tachycardia. They cannot communicate their symptoms clearly, and if prolonged attacks are not recognized they may develop signs of heart failure before receiving appropriate treatment.

The condition is uncommon at that age. Several large clinics on both sides of the Atlantic have reported seeing only two or three cases a year. ${ }^{1-4}$ But these tend to be the serious cases, and its prevalence in the community is probably much higher than such reports suggest. Many relatively short-lived and isolated attacks revert spontaneously to normal rhythm and pass unnoticed. Moreover, not all of those detected are referred to hospitals for treatment, and some that are never reach the cardiac clinic.

Though all types of rapid dysrhythmias are encountered in young children, most are supraventricular. Paroxysmal atrial tachycardia is much the most common. Atrial flutter is seen less often and atrial fibrillation only occasionally. Ventricular tachycardia is rare unless associated with severe myocardial disease. Only between a quarter and a third of those whose paroxysms are treated in hospital have congenital or acquired heart disease. If they are excluded, it can be said with confidence of the remainder that most attacks occur in children with otherwise healthy hearts and that the paroxysmal tachycardia itself has no sinister significance. In some cases it is occasioned by intercurrent infections such as pneumonia, meningitis, otitis media, or myocarditis, but an obvious cause is the exception rather than the rule. A special group are children with the WolffParkinson-White syndrome block who can readily be identified by electrocardiographic evidence of ventricular preexcitation. They are likely to have recurrent paroxysms that are often difficult to control.

So far as age is concerned, children with paroxysmal tachycardia can be divided into two groups. The first and largest is a group of infants whose paroxysms nearly always occur without obvious cause in the first few weeks or months of life and who are nearly all boys. They tend to have a better prognosis than the second group, whose paroxysms occur later in life, are scattered throughout their childhood years, and are more likely to recur than those that begin in infancy. The older children, too, or their parents are usually aware of rapid heart action, palpitation, or even precordial constriction and seldom present much diagnostic difficulty. The tachycardia is therefore unlikely to be overlooked or to persist long enough to cause circulatory embarrassment. But babies and small children often present as emergencies without the heart coming under suspicion. They appear ashen grey or perhaps a little cyanosed, cold and sweating, with rapid, laboured respiration and cough because of left ventricular failure. Both the heart and liver may be enlarged. The heart rate is usually well over 180 and is frequently nearer 300 per minute. At such speeds it is impossible to count accurately without electrocardiographic control and all that can be said clinically is that it is extremely fast. The fact that an infant's heart rate is normally in the region of 140-150 during the first few months of life, and with sinus tachycardia may approach 200 , often diverts attention from the cardiovascular system as a primary source of the acute illness. But the rate of the paroxysm is generally less important than its length. The heart seldom fails unless it has persisted for at least 24 hours, and failure becomes more and more likely after 48 hours.

When assessing the likelihood of recurrence, a clear distinction must be made between those children who have ventricular pre-excitation and those who do not. In a recent follow-up study, which is in broad agreement with most previously published work, A. Lundberg ${ }^{5}$ has shown that the chance of recurrence in infancy is $65 \%$ with pre-excitation and $46 \%$ without it, while between the ages of 1 and 10 years the comparable figures are $46 \%$ and $6 \%$. Only exceptionally are recurrent attacks anything other than a minor inconvenience in older children, and in the absence of organic heart disease the long-term prognosis is usually excellent.

In treatment one must differentiate between stopping the paroxysms and preventing their recurrence. Digoxin is still the drug of choice for the treatment of attacks, especially if signs of heart failure are present. Direct-current shock is the quickest and most effective way of terminating an attack, but is suitable only for hospital treatment. Safe and simple, it is probably used less often than it should be in paediatrics. Certainly it should be considered early in the list of alternatives and not as a last resort when all else has failed. The ideal antidysrhythmic drug has still to be found. Recently, beta adrenergic blockers have been more promising than most and should now be considered among the first options.

In all but a few patients the paroxysmal supraventricular tachycardia can be terminated, and rare cases of chronic tachycardia, which may be either persistent or repetitive over months or years, ${ }^{67}$ can usually be controlled. In these chronic cases the rapid heant rate is usually slower than in acute paroxysms and is well tolerated, so that even in the most resistant cases the prognosis is not necessarily serious, and many will revert sooner or later to normal sinus rhythm.8

While most paroxysms can be successfully terminated, the same success cannot be claimed fior their prevention. Fortunately for the majority of patients prophylactic therapy is unnecessary. Their attacks are infrequent and can easily be dealt with by physical or chemical methods as they occur. Babies are the exception and should be carefully supervised, because diagnosis is difficult and the risk of heart failure is high. A good case could be made for maintenance digoxin in all of them. It should certainly be prescribed for those with pre-excitation until they are old enough to communicate.

For those whose attacks are frequent enough to warrant continuous drug maintenance, digoxin is again the drug of choice. If this is ineffective, a beta-adrenergic blocker such as propranolol should be tried either alone or in combination with the digoxin. When this fails to control recurrences, the chances of successful control are poor. The old practice of adding quinidine when digoxin has failed to terminate or prevent paroxysms cannot be recommended. Quinidine is a dangerous drug, probably much more dangerous than is generally appreciated..$^{10}$ It has been under suspicion for a long time as a cause of sudden death, often after relatively small doses. Recent evidence confirms that it should be used only as a last resort and if treatment is really essential. The fact that procainamide has become the most frequent cause of drug-induced lupus erythematosus ${ }^{11} 12$ further limits the choice of effective drugs available. It should be given only on a short-term basis because antinuclear antibodies are found in at least $50 \%$ of patients after pro- 
longed treatment. Lupus erythematosus may develop in about $20 \%$ of them and does not always pass into remission when treatment is discontinued. Many other drugs have been used in attempts to suppress recurrent paroxysms; verapamil is a recent example. ${ }^{13}$ Sometimes they are successful, but like coronary antery surgery, treatment tends to be most effective where it is least required and least effective where it is most required.

Though physical exertion may sometimes trigger an attack, children with paroxysmal supraventricular tachycardia should be encouraged to live normal lives. No restrictions should be placed on their activities either at home or school. Above all, they must be taught to regard the attacks as a minor inconvenience and not as evidence of heart disease.

\footnotetext{
1 Nadas, A. S., Daeschner, C. W., Roth, A., and Blumenthal, S. L., Pediatrics, 1952, 9, 167.

2 Simcha, A., and Bonham-Carter, R. E., Lancet, 1971, 1, 832.

3 Klint, R. B., Hernandez, A., Goldring, D., and Behrer, R. M., Clinical Pediatrics, 1972, 11, 475.

Dworkin, P. H., Bell, B. B., and Mirowski, M., Archives of Diseases in Childhood, 1973, 48, 382.

Lundberg, A., Pediatrics, 1973, 51, 26.

Hay, J. D., and Keidan, S. E., British Heart Fournal, 1952, 14, 345.

Keane, J. F., Plauth, W. H., jun., and Nadas, A. S., American Heart fournal, 1972, 84, 748.

8 Zetterqvist, P., Bulletin of the Association of European Paediatric Cardiologists, $1972,8,42$.

- British Medical fournal, 1969, 1, 331.

10 Lindsay, J., jun., American Heart fournal, 1973, 85, 141.

11 Condemi, J. J., Blomgren, S. E., and Vaughan, J. H., Bulletin on the Rheumatic Diseases, 1970, $20,604$.

12 Kosowsky, B. D., Taylor, J., Lown, B., and Ritchie, R. F., Circulation, 1973, 47, 1204.

${ }^{13}$ Husaini, M. H., Kvasnicka, J., Ryden, L., and Holmberg, S., British Heart fournal, 1973, 35, 734.
}

\section{Scleroderma or Sclerodermas?}

The name scleroderma describes several apparently distinct syndromes in which sclerosis of the skin is prominent. But as is almost inevitable when classification is based on appearances rather than an understanding of the aetiology intermediate cases occur which seem only to confuse our ideas. The similar names of several other entirely separate diseases add to the confusion.

Scleroderma is usually subdivided into localized, or morphoea, and generalized, better called systemic sclerosis. Morphoea typically presents with round or oval plaques of sclerosis with a violaceous edge, but linear lesions are fairly common. It is supposed not to lead on to systemic disease, but the process can spread to the subjacent connective tissue, muscle, and even bone. The linear lesions are especially apt to do this, and widespread or localized arthralgia has occurred in up to $40 \%$ of cases. ${ }^{1}$ Electronmicroscopy shows that subclinical lesions of muscle are rather frequent. ${ }^{2} 3$ In some cases atrophy rather than sclerosis of the skin and underlying tissues may dominate the picture.

The concept of generalized morphoea and its relationship to systemic sclerosis has vexed dermatologists for many years. It is generally agreed that morphoea can be very widespread, often in places retaining its violaceous edge, and can even progress to affect the whole skin. ${ }^{5}$ The prognosis for life in such cases is fairly good, and the skin changes may show partial or complete regression over some years. But at a single examination these cases may be indistinguishable from a rare type of systemic sclerosis without involve- ment of the extremities. In contrast, cases of unequivocal systemic sclerosis can show plaques of morphoea.

The typical case of systemic sclerosis presents with slowly progressive Raynaud's syndrome, sclerosis of the fingers (sclerodactyly), and of other extremities, so that the name acrosclerosis is often used. lesions in other organs, notably the oesophagus, small and large intestine, kidneys, lungs, heart, eyes, and joints are usual but not all in one patient. But not all cases of systemic sclerosis have Raynaud's syndrome or an acral distribution of sclerosis. In some the skin is spared altogether. Others shows extensive sclerosis of the skin of rapid onset, and often the prognosis is poor owing to early involvement of other organs. ${ }^{6}$ These rare cases have caused confusion with generalized morphoea.

The aetiology of scleroderma is unknown. ${ }^{7}$ The lightmicroscopic and electron-microscopic changes of systemic sclerosis and morphoea are essentially similar, though some workers have found slight differences-for example, in the lesions of small blood vessels. 9 Though the most prominent change is often an abnormality and coarseness of the collagen fibres in the dermis, the total collagen may not be increased. ${ }^{10}$ Early changes can be found in the subcutaneous tissue, ${ }^{2} 3$ and the sclerosis may be partly due to an abnormal architecture of the collagen, with a tethering of underlying tissues or to abnormal forces binding the individual fibres together. ${ }^{11}$

The nervous system probably plays some part in scleroderma of all types. ${ }^{12}$ Whether primary Raynaud's disease without evidence of scleroderma can be considered a forme fruste of scleroderma is uncertain, but patients can be reassured that if the syndrome has lasted more than two years without evidence of scleroderma the chances of its developing are small, though not excluded. ${ }^{13}$

The evidence linking scleroderma with other so-called connective tissue diseases and for its having an autoimmune aetiology is equivocal. About one-third of cases of systemic sclerosis have joint changes, but biopsy shows them to be different from rheumatoid arthritis, ${ }^{14}$ and a variety of pseudosclerodermatous conditions can occur with rheumatoid arthritis. ${ }^{12}$ However, a true association of scleroderma, rheumatoid arthritis, dermatomyositis, and lupus erythematosus does occur. ${ }^{15} \cdot{ }^{16} \mathrm{E}$. L. Dubois and his colleagues ${ }^{17}$ have also described cases of systemic sclerosis and even of morphoea with features resembling systemic lupus erythematosus. These cases are important for our understanding of the diseases and also because the patients respond better to corticosteroid therapy than can be expected with pure scleroderma.

Sclerosis of the skin can also occur without the sinister implications of systemic involvement. The commonest of these syndromes is the sclerosis of the lower legs which some patients experience in association with venous stasis. A similar picture can occur in scurvy in the absence of the better known follicular haemorrhages. ${ }^{18}$

The relationship of lichen sclerosus to morphoea is uncertain. Werner's syndrome, porphyria cutanea tarda, and the confusingly named sclerema, scleroedema, and scleromyxoedema are all apparently separate disorders, though they sometimes show sclerosis of the skin. There have been four reports, of which the most recent is that of J. F. Fries and colleagues, ${ }^{19}$ of skin changes clinically resembling widespread scleroderma but without Raynaud's syndrome, associated with a secondary carcinoid tumour. Other organs are not affected. How this tumour leads to sclerosis is not clear, 